\title{
Leprosy and the rhetoric of elimination
}

In this Feature in the following sentence, the word "not" is missing (BMJ 2013;347:f6142, doi:10.1136/bmj.f6142). The sentence should have read: "Only two states-Bihar and Chhattisgarh - and one union territory-Dadra and Nagar Haveli-have not declared elimination of leprosy as a public health problem, but the sample survey, which has been seen by the $B M J$, found that rates in some states were several times higher than the reported figures.'

Cite this as: BMJ 2013;347:f6717

๑ B BMJ Publishing Group Ltd 2013 